\title{
Information architecture for social media: a case study on building an event backchannel with Twitter
}

\author{
Robert L. Moore \\ School of Government, \\ University of North Carolina at Chapel Hill, \\ Knapp-Sanders Building, CB 3330, \\ Chapel Hill, NC 27514, USA \\ E-mail:rob@mindofaninnovator.com
}

\begin{abstract}
This paper presents a case study on creating a backchannel through Twitter for the live event, featuring the Secretary of the US Navy, hosted by the School of Government. The project, which ultimately was successful in creating social media buzz for the lecture, was a new approach for the School of Government in how it markets its events. The study discusses the tools and processes used in the backchannel's creation and development. This paper was written because the author discovered a gap in existing literature on creating backchannels. It outlines how the adaptation of best practices from the general population's use of Twitter in creating a backchannel for an educational event. While this study focuses specifically on the promotion of a live event, the concepts and principles discussed here also are applicable to instructors interested in providing their students with an environment for differentiated learning and informal communication.
\end{abstract}

Keywords: social media; information architecture; backchannel; Twitter; case study; best practices; analytics; social media analytics; engagement; social media engagement; interactive learning environments; differentiated learning; informal communication; event backchannel.

Reference to this paper should be made as follows: Moore, R.L. (2014) 'Information architecture for social media: a case study on building an event backchannel with Twitter', Int. J. Social Media and Interactive Learning Environments, Vol. 2, No. 1, pp.21-36.

Biographical notes: Robert L. Moore is a Lead Instructional Designer with the University of North Carolina at Chapel Hill School of Government and has worked at UNC since 2004. He joined the Instructional Support team in September 2010 after spending five years managing the UNC Foreign Language Resource Center. In his current position, he collaborates with faculty on integrating innovative technology to support their instruction in face-to-face, blended, and online instructional environments. He holds an MS in Instructional Technology from the East Carolina University and a BA in Political Science from the UNC-Chapel Hill and is a UNC-Chapel Hill Center for Faculty Excellence Future Faculty Fellow. He has also attained the LEARN NC Online Instructor certification and the ECU Distance Learning and Administration certificate. 


\section{Introduction}

In August 2012, the Marketing and Communications Division and the MPA Program at the UNC School of Government (SOG) decided that the November 1, 2012, Deil S. Wright lecture would feature Secretary of the US Navy, Ray Mabus. The Marketing and Communications Divisions determined that the lecture, hosted by the Master of Administration (MPA) program and sponsored by Fidelity Investments and the MPA Alumni Association, needed social media buzz. Event organisers were interested in ways that social media could help extend the awareness of the upcoming lecture beyond just the participants in the MPA Annual Conference that was also held that weekend. Although Marketing and Communications had expertise with traditional marketing practices, they had not previously used social media for an event. Thus, they reached out to the School's Instructional Support team, specifically, the author, for assistance in incorporating social media into their event. The author is an instructional designer on the Instructional Support team and regularly collaborates with faculty and staff interested in integrated technology into their instruction.

At any live event, attendees can be found communicating about what is currently happening, and collectively, these communications are referred to as a backchannel. By using backchannels event organisers can tap into real-time feedback and incorporate comments and questions into the event to make it more responsive and dynamic. Information architects Morville and Rosenfield (2002, p.5) state that "knowledge managers develop tools, processes and incentives to encourage people to share [information]", and just as information architects plan the different design layers of large-scale websites, a social media backchannel has its own building process. And while there have been a few studies seeking to gauge the effectiveness of a given backchannel, the author noticed a gap in the scholarly research explaining how to create a backchannel. The author believed that an understanding of the core concepts of Twitter and of Twitter engagement could be used to effectively develop backchannel-creation steps. This case study provides an overview of these concepts and outlines the way they were adapted to create the steps.

This study is organised into three sections. The first section provides an overview and introduction to Twitter and backchannels and gives some context for why Twitter can be an effective way to develop an event backchannel. The second section details the specific event - a lecture by the Secretary of the US Navy. The final portion of the case study looks at ways to improve the backchannel/social media process for future SOG events as well as for other purposes such as the MPA graduation or promotions around new publications.

\section{Overview of Twitter and backchannels}

\subsection{What is Twitter?}

\subsubsection{Generally}

Twitter is a quick, easy way for people to share their thoughts, or tweets, on any range of topics and create impromptu communication networks. Ebner et al. (2010) posit that Twitter may be changing the way we communicate with each other and the way we share 
information. It has rapidly become a centralised location for finding out what is happening in the world. Qualman (2013, p.10) points out that "we no longer search for the news; rather the news finds us". The news that 'finds us' often looks different than it used to: today we see broadcast media outlets trying to engage viewers through social media in ways that were not done in the past, in part because advertisers are able to get a higher return on investment (ROI) when they use social media versus traditional marketing approaches (Qualman, 2013).

A contributing factor to this marketing shift is an increase in the use of DVR and TiVo devices that allow viewers to fast forward through television commercials. The increased popularity of these devices explains why most channels that offer their shows on-demand do not allow viewers to fast forward through commercials and why, when you visit YouTube, Hulu, or other online video services, you have to watch the ads. Not too long ago, the highlight of the Super Bowl was the ads. Not only are the best commercials available online, television shows, regardless of genre are also attempting to encourage viewers to actively engage with each other in real-time via Twitter. Advertisements gain the attention of viewers by featuring text such as 'Follow us@[showname]' or 'Join the conversation at \#[showname]' on the screen during the show. Companies responsible for developing marketing campaigns understood that people were not going to be watching the commercials on television but were in the digital space. They realised that they needed to put the ads where the viewers were not only most likely to see them but also most likely to engage with the product. Shifting of the focus to online mediums allows viewers to continue to engage with one other even after the event/show has concluded, which is one reason that Harrington et al. (2012) suggest that Twitter is best viewed as a complementary type of media channel.

For the Mabus lecture, the event organisers wanted to use traditional press releases and media advisories. To complement these efforts and to integrate social media, the event hashtag (a Twitter tagging action that solicits comments) was added to the flyer published in the campus newspaper. By encouraging the use of specific hashtags, consumers of certain products or events are able to reach the entire community of users (Highfield et al., 2013), and this can extend the reach of the product or event. Building and connecting groups of people around a common hashtag is the core concept of a backchannel and it can be used to great effect by event organisers.

\subsubsection{Specifically}

Twitter is the social network that allows users to engage and interact with these tweets and the act of tweeting is termed microblogging. To get started on Twitter, one creates a Twitter handle or username at http://www.twitter.com in the form of @[yourusername]. Creating an account enables people to 'follow' you and read your 140-character messages called tweets. As with most social networks, you choose whom you wish to follow by searching for the particular person or entity and then selecting the 'Follow' button on the profile page You also have the ability to 'unfollow' someone, by selecting 'Unfollow' on their profile page.

Twitter conversations begin with 'mentioning' another user and may take one of the following forms: 
1 replies to posts made by people your followers

2 replies made by your followers to posts by other Twitter users; because someone you follow is involved, that conversation gets added to your Twitter stream

3 organic tweets generated by a user, incorporating 'trending' topics (those showing increased popularity) and selective engagement, with the topics/users that interest you.

To interact with specific Twitter users, you add their Twitter handles to the @ symbol (e.g., ‘@Starbucks'). Anyone can also retweet (or RT) your messages to his or her own followers.

Qualman (2013, p.18) identifies the "key aspect of social media [as] the ability to tag items". On Twitter, this tagging of items is in the form of a hashtag - as represented by the \# symbol - combined with a phrase or keyword that is searchable or indexable. For instance, the hashtag '\#PolandNeedsAOneDirectionConcert' represents a trending topic of a group of Twitter users, likely in or near Poland, that are interested in having a One Direction concert. Just as the hashtag allows you to filter through tweets to find what interests you, it can also be used to filter out tweets that do not. Recently released movies use hashtags to build buzz, for example, '\#TDKR' (for the final Batman movie, 'The Dark Knight Rises'). Twitter users who have watched the movie share their thoughts and use the hashtag to let their followers understand what they are talking about but also to allow for non-followers to be able to see their tweets if they search for the hashtag. Those who have not seen the movie yet and do not want any spoilers can mute the hashtag which is another benefit of using a hashtag. Just as hashtags allow one to index their tweets, it also allows others the ability to hide or filter your tweets. This function is available in the different Twitter applications used to access your Twitter stream. In this example, once you have seen the movie, you can unmute the hashtag and begin to interact with those discussing it. In addition to being searchable within the Twitter application itself, tweets are also indexed by search engines (McNely, 2009). Thus, one can run the Twitter search '\#Scandal' and find all of the tweets about the ABC hit drama 'Scandal' as well as run a Google search for 'Scandal ABC' and find tweets included in the list of search results, among other sources.

Imagine going to a local sports bar alone to watch your favourite team play. During the game, you react, comment and interact with others at the bar. You may make enemies with fans of the other team or new friends with others who support your team. You walked into the bar by yourself, but you found some common ground to enjoy the game with others. Now, multiply those comments about the game at the bar 100 times - that is the Twitter experience. Twitter allows people around the world to see, react to and engage with one another even if they are in disparate locations. More specifically, by enabling anyone to send a tweet about from anywhere whether it is via text, mobile or desktop application, the ability for real-time updates is not only possible but a reality of today's media awareness and this makes information move at a much faster rate.

Perhaps most importantly, Twitter allows anyone to become an information creator. As Bakshy et al. (2011) state, "Everyone's an Influencer". So instead of being a mere consumer, anyone can get his or her message out to the masses. It is not uncommon to see a news station ask its followers to share pictures from a big snowstorm or other local event by using a hashtag. McNely (2009) outlines seven reasons why Twitter is such a successful microblogging system: "low barriers to participation; SMS functionality 
[meaning short message service, such as text messaging]; simple syntax; asymmetric social relationships; aggregation; distribution; and real-time delivery" (p.302). Murthy (2011) concurs, attributing the similarity between Twitter and texting to a low barrier of entry and use.

A social media power user - one who tweets and engages with social media on a regular basis - normally uses an application capable of running on his or her internet-enabled smartphone or desktop. For desktop users, http://www.tweetdeck.com and http://www.hootsuite.com are two of the more popular Twitter applications. These applications, referred to as third-party aggregators, allow you to use one application to access and manage multiple social media accounts, from Facebook to LinkedIn to Twitter; this is considered a huge time saver.

With Twitter particularly, these kinds of applications can help organise and manage one's Twitter experience by allowing for the creation of custom searches or lists of different Twitter handles. Both TweetDeck and HootSuite allow you to create a column within the application that displays these custom searches or tweets from users within your list as shown in Figure 1. Additionally, TweetDeck and Hootsuite allow for multiple columns, which is not something that can be done if using the web-based version of Twitter. These columns are especially useful when managing a backchannel. Another example is that one could create a column for the hashtag of your favourite show: '\#Scandal'. This would allow you to connect with people you did not previously follow but with whom you share an interest in the same show.

Figure 1 Screenshot of TweetDeck columns (see online version for colours)

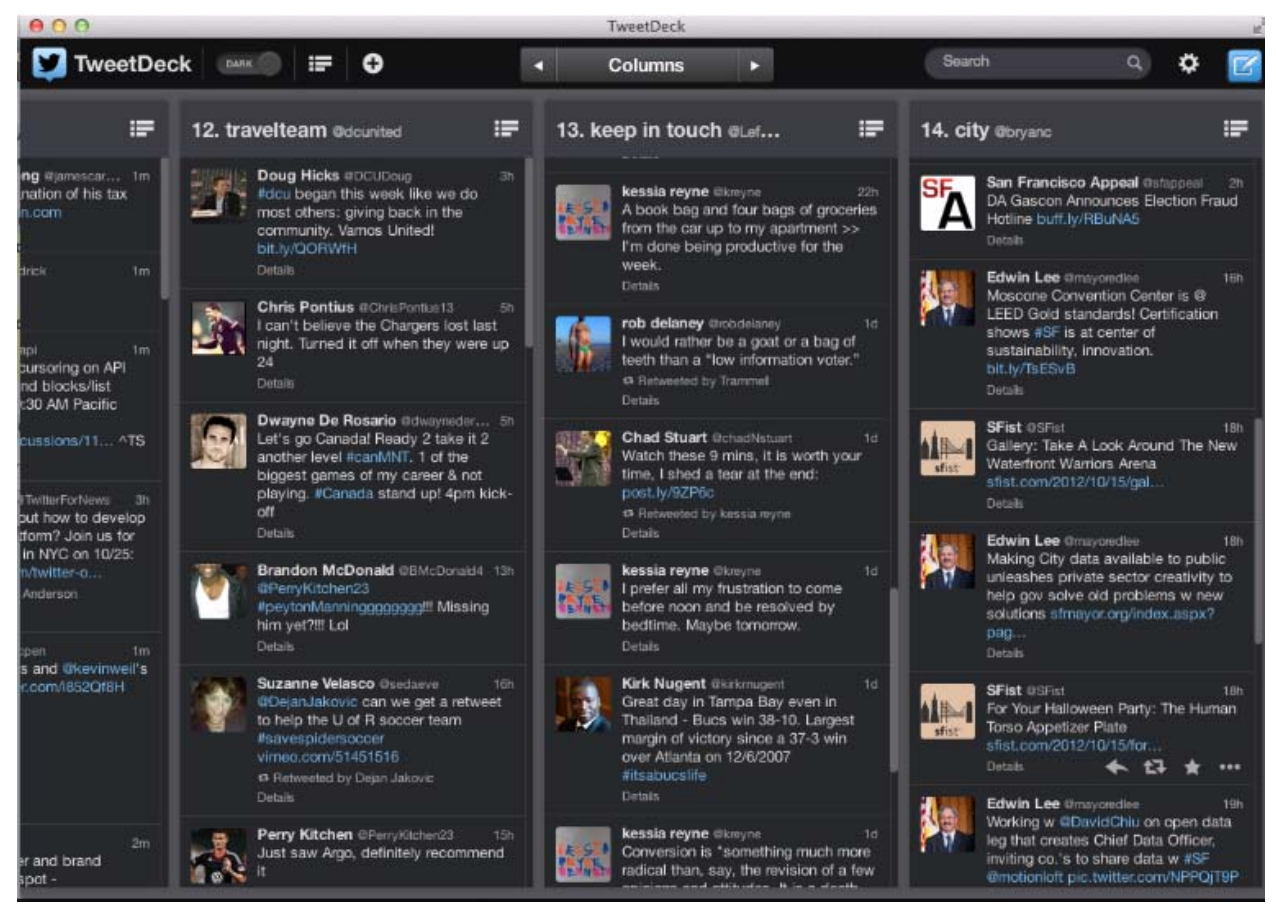

Source: https://support.twitter.com/articles/ 20169620-getting-started-with-tweetdeck. 
Lists are an effective way to manage specific groups of people. For instance, you could make a list of your favourite athletes. By doing this, you can find their tweets more easily since they are all displayed in a single column. Such techniques are helpful for indexing and filtering through the incredible amount of information put out via tweets. And just as these techniques are useful for tracking conversations from athletes or discussions about a favourite TV show, Ferriter (2010) points out that they could be similarly used by educators looking to establish, develop, and engage within their own personal learning networks.

\subsection{What is a backchannel?}

Think back to a time when you were at a movie or a lecture and quietly said something to your neighbour about the event. Now, imagine those thoughts being tweeted to many people instead of whispered to just one person, and then imagine others with the same thoughts commenting on your original post. This communication mix is what is called a backchannel. As technology has improved, the creation of backchannels has become the norm not only at conferences, but also within organisations (Ross et al., 2011). Backchannels are seen not as a way to replace a speaker or conference but, rather, as a way to supplement and bolster participation within the event itself. As McNely (2009) states, a backchannel's main purpose is to provide a "secondary or background complement to an existing frontchannel" (p.297).

In some cases, backchannels provide more interaction between an event's speaker and participants. Backchannels move conversations and thoughts from just one or two people to an entire group. In the words of Ross et al. (2011), a backchannel can "... [change] the dynamics of the lecture room from a one to many transmission to a many to many interaction, without disrupting the main channel communication" (p.215). A backchannel also enables someone not physically present at an event to be able to participate in conversations about it. This is particularly useful with lectures, conferences or other one-time live events. Though they do not replace the advantages of attending the event, backchannels are an invaluable resource for those who are constrained by tight travel budgets and time commitments as they provide real-time summaries or posts of the most salient points from a live event. According to McNely (2009), one of the main challenges with non-digital backchannels (e.g., the side conversation that a lecture attendee has with someone sitting next to him or her during an event) was the lack of persistence of the backchannel - and the inability to preserve this non-digital backchannel for later research and analysis. This side conversation existed orally and involved only the two people who were conversing; if others in the lecture had similar thoughts, no one would know since they were not captured in a digital or shareable format. Twitter does not suffer from this deficiency; in fact, its persistence is one of its strengths, which is further augmented with the use of a conference hashtag to facilitate the curation and organisation of backchannel communications. Although Web 2.0 tools like Twitter are easy to use and have low barriers of access and deployment, a successful backchannel nonetheless requires careful planning. 


\section{Description of project}

To promote the Mabus lecture, the SOG Marketing and Communications Division employed traditional media tactics, such as drafting press releases for media advisories; communicating with news media; and facilitating print advertisements for the event, such as ads in the university newspaper, the Daily Tar Heel, and event flyers. To supplement these efforts with social media tools, the author worked with an MPA graduate student and a federal work - study student. The goal was to spread the word about the event as widely as possible. Creating a backchannel was selected as the way to best achieve this goal because it would provide some quantifiable evidence of the social media buzz for one of its co-sponsors, Fidelity Investments, in addition to remote participation.

Based on the conclusions of Ebner et al. (2010), even though the event organisers knew that remote participation would be low and have minimal impact, they nonetheless felt that the social media tools and techniques used to create the backchannel would aid in the creation of the desired social media buzz. In the next section, the author explains the specific steps used in the case study and these steps are depicted in Figure 2.

Figure 2 Steps used by SOG to create a twitter backchannel (see online version for colours)

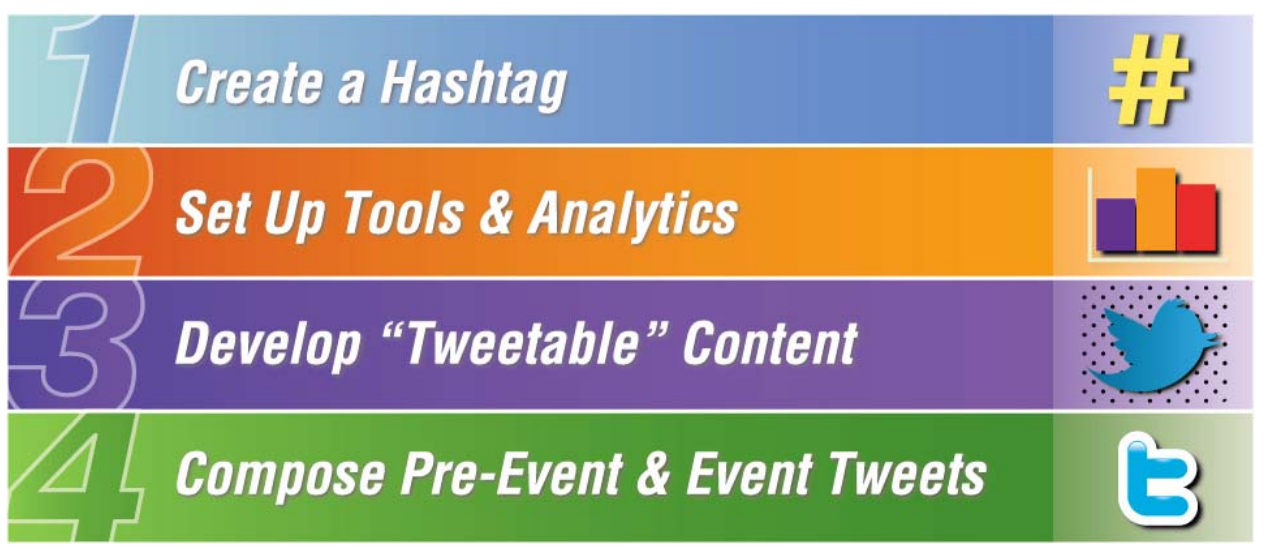

Source: Author

\section{Backchannel process}

\subsection{Step 1: Create a hashtag}

Social media is easy to use, making it one of the more open and flexible communication mediums available. This is both advantageous and challenging: since it is so user-friendly, you experience a lot of social media traffic or noise, meaning a lot of information of varying types, gets put out through social media channels. There is a common misconception that Twitter and Facebook status updates are comprised entirely of posts about what users ate for lunch. While people do write about such things, there is also a great deal more substantive information posted through social media channels. The sheer volume of daily tweets can be overwhelming, particularly to someone new or unfamiliar with the medium. One of the ways Twitter addresses problems presented by 
this much information, is through the ability to index and search tweets, actions which are most easily accomplished through the use of hashtags. An effective way to filter conversations and information, hashtag use is enhanced by precise selection. Thus, selecting a hashtag early in the process is crucial, as it will serve as a tagging mechanism for future social media uses.

\subsubsection{Creating \#uncmabus}

For the MPA lecture, a hashtag was created for the backchannel. To come up with the right hashtag, a search of different terms, such as Mabus, MPA, etc., was done at http://search.twitter.com to see what was already in use. A search was also done for 'Secretary of the Navy' and similar terms to see how Secretary Mabus was referenced on social media. After analysing the search results, it was determined that '\#uncmabus' would be the best hashtag because it was short enough to be easily included in tweets without taking up too many of the allotted 140 characters and it was very clear that it concerned the Secretary of the Navy, Mabus, and UNC. The next step was registering the hashtag at http://www.twubs.com. Twubs allows each registered hashtag to carry an explanatory description, which is useful for those interested in learning why or how the hashtag will be used. It is important to register a hashtag because doing so ensures that it will not be duplicated and makes it easier for people to find the hashtag when doing a search.

At this point, we now had a specific hashtag for the event, \#uncmabus, a twitter account, @UNCMPA (established before this case study), and a webpage on the SOG website (http://sog.unc.edu/node/1971, also already established) for information about the event. Additionally, the MPA program had an existing Facebook page, which was also used for posting information about the event.

\subsection{Step 2: Set up tools and analytics}

In this stage, event organisers need to decide what types of information they would like to have tracked, such as 'hit count' (how many people visited on the event site), number of followers gained or number of clicks for a specific link. The author strongly recommends that organisers make use of a third-party aggregator, such as HootSuite or TweetDeck. These aggregators can be run on a desktop computer and offer many features that will facilitate the management of multiple social media accounts and allow for efficient cross-posting to various social media channels. Also, the efficiency of being able to use one programme to post to multiple social media channels can greatly aid in developing and managing the backchannel. Each of these aggregators offers specific features and may require some experimentation to find the one that best fits your needs. For instance, HootSuite is ideal when there is one account being managed by multiple people, but it is a less than ideal choice of aggregator when people are interested in managing both their personal and shared professional accounts. In this latter case, TweetDeck would be the better choice, as it allows for shared professional accounts and personal accounts managed through different installations.

Analytics should also be considered at this phase of the backchannel process. In the social media context, analytics refers to how one can quantify the success, or lack thereof, of a social media campaign. This is done through an analysis of the tweets, mentions, and RTs in an attempt to assess the number of people that saw and 
engaged with your information or content. This analysis reveals what is trending - which tweets are getting the most traction (or mentions and RTs) on Twitter or other social networks.

Social media occurs in real-time and is dynamic and constantly evolving. Analytics can help identify trends, allowing you to adapt your strategy in real-time and thus serves as a form of formative assessment. Event organisers should review different sites offering analytics of social media accounts and align a given site's features with the specific needs of their projects. There are some free sites that may provide adequate enough information, but certain project needs may call for the use of a paid or subscription-based site.

\subsubsection{Creation of bit.ly/uncmabus: using TweetDeck}

The website URL for the lecture informational page created on the SOG website was long and difficult to remember. Using it would limit the amount of sharing that could happen on social media channels. Also, many social media tools would likely auto-shorten the URL, rendering event organisers unable to track the number of clicks on the link, thereby making it difficult to evaluate whether or not the social media efforts had generated any type of traffic to the informational page. The easy solution for this was to use the bit.ly URL shortening service (see http:/www.bit.ly). Bit.ly was chosen because it provided an easy-to-use, web-based analytics interface. Since the event hashtag was already selected, the informational webpage was converted from http://www.sog.unc.edu/node/1971 (32 characters) to http://bit.ly/uncmabus (22 characters). This link was added to tweets and RTs and used on Facebook posts. It allowed for easier sharing and sent people to the informational webpage maintained by the Marketing and Communications Division. Over the course of the month in which the event and its promotion took place, this link was accessed about 140 times (see Figure 3), with the traffic coming primarily from Twitter (see Figure 4).

Figure 3 Clicks on the uncmabus bit.ly link, October 6-November 6, 2012

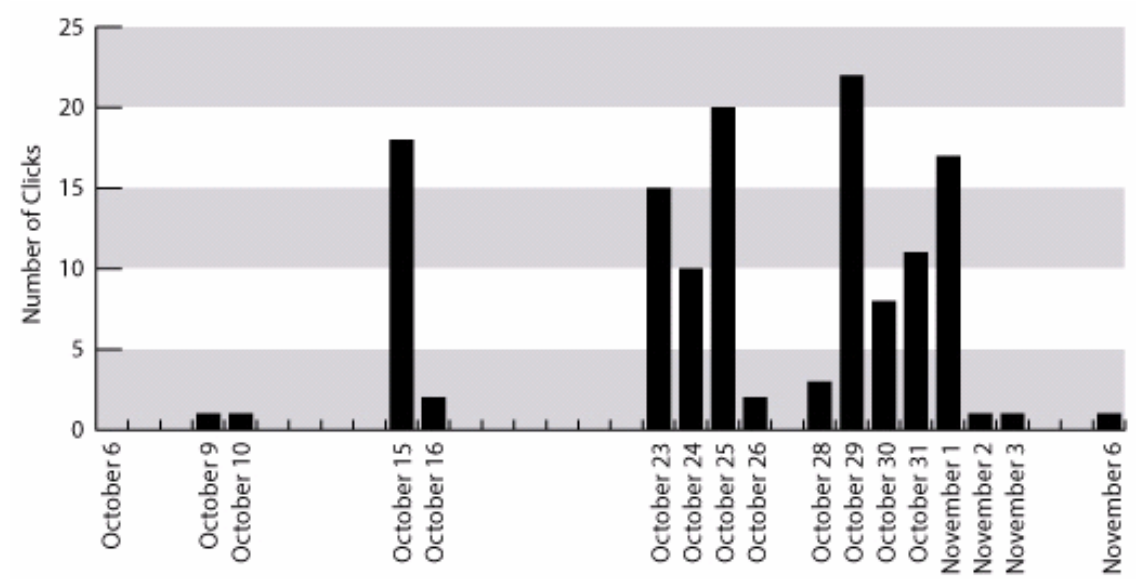

Source: http://bit.ly/uncmabus+/global 
Figure 4 Referrers for clicks of the uncmabus bit.ly link, October 6-November 6, 2012

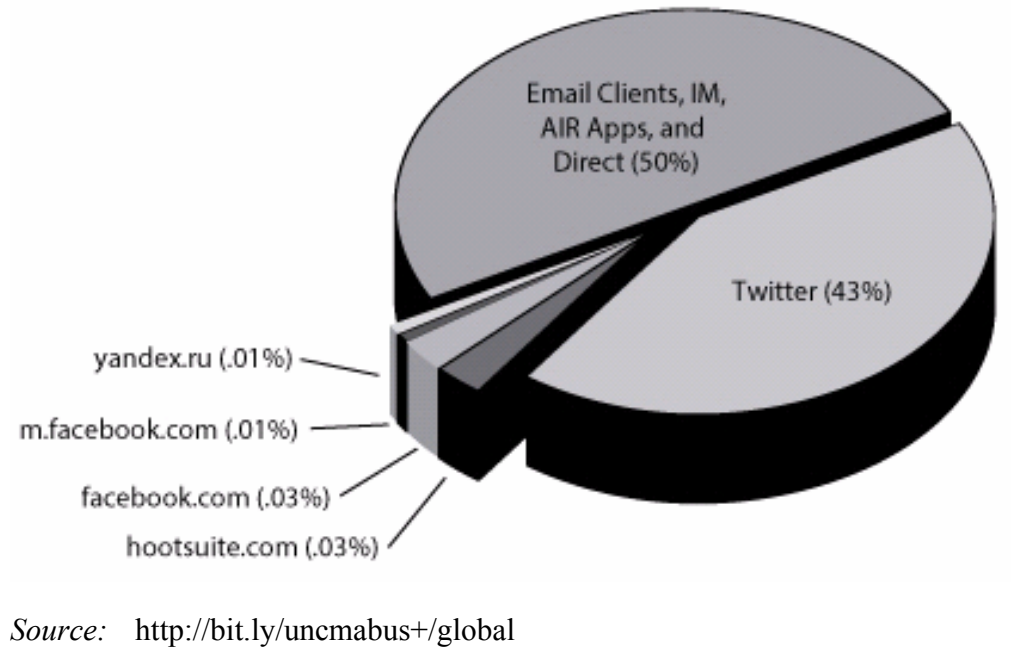

This information about clicks was helpful in tracking data for event organisers who knew that the only people seeing the bit.ly link were doing so through social media channels or as a result of direct e-mails. As the lecture date neared, organisers sent out e-mails to different campus contacts asking them to post and share event information. The bit.ly link made it easier to post those messages, and the click-through rate showed that people were clicking on the link in the e-mails.

Columns were created within TweetDeck for the monitoring of search terms such as 'Ray Mabus' and also for keeping track of the event hashtag itself. Since all of this information showed up in its own column, it was easy to track tweets and identify new information. For instance, an interesting tweet about Ray Mabus could be retweeted using the hashtag to help build awareness about the Secretary's upcoming UNC lecture. Through TweetDeck, event organisers were able to find other accounts that were tweeting about the Secretary which enabled them to send out additional tweets to specific people who might be interested in his speech at UNC. The columns also allowed organisers to search relevant terms and catch tweets that might belong on the backchannel but were missing from it. These missing tweets were then retweeted with the event hashtag to move them into the backchannel.

Crowdbooster $($ was selected for the analytics portion of the tweeting because, at the time, it provided a free account with robust reporting features. Since the event, however, the service has moved to a paid, by-subscription model. Crowdbooster was used in addition to the bit.ly tracking of the specific URL (discussed above). A Crowdbooster account was created for the main twitter account, @UNCMPA, and this service looked at the number of tweets and calculated the number of impressions each tweet was receiving. As Crowdbooster.com explains, an impression is "an estimate of the total possible number of times someone could have seen your tweet." The site continues its explanation by noting that these impressions are "the sum of your followers and the followers of your retweeters for a particular tweet" (https://crowdbooster.com/faqs/). The event organisers knew that they would need to provide a final report of their successes on this project to the sponsors of the event, specifically, to Fidelity Investments, and they felt that 
Crowdbooster would help provide statistics that could be easily understood, even by non-social media users.

\subsection{Step 3: Develop 'Tweetable' content}

At this point different social media accounts have been created as has an informational webpage or website using a bit.ly link, and it is now time to start generating and developing information to share through these different social media accounts. It is now important to determine what types of messages you will send. Think about why someone would be interested in the event and then decide how to best reach that person. Will you be posting videos? Do you have information about the event or the speaker that is especially helpful or interesting? To help in the development of the content, it is useful to think of other people or accounts that may assist in the creation and sharing of event-related information. Creating a 'talking points' document can be a helpful and effective way to coordinate the development of a backchannel when one wants to have multiple people tweeting to build awareness of both the backchannel and the event itself. This document acts as a style guide, providing consistent guidelines for tweets, such as which accounts to reference, what type of information to focus on or include, and suggested tweets.

Event organisers are advised to choose information that comes in a 'tweetable' format, meaning content that uses the appropriate hashtags and usernames and contains approximately 100 characters which Salesforce Marketing Cloud (2012) reports can generate a $17 \%$ higher rate of engagement on Twitter (p.11). A tweet can only be 140 characters long, including username, but setting a 100-character ceiling allows the inclusion of the original tweeter's username as well as your own in the RT. This, along with the creation of a style guide, will make it easier for different people to post messages from their own, personal accounts. All of this will start to build traffic for the hashtag and jumpstart the buzz. Also, since the social media information cycle moves so quickly, one will want to wait until the event date is near before doing the bulk of the event tweeting. The frequency of tweets is another consideration. Salesforce Market Cloud (2012) would recommend that the goal should be to put out no more than four tweets per day spread out over different times during the day for maximum effectiveness.

\subsubsection{Development of the 'talking points' document}

'Leadership in Service' Was the topic for the Mabus lecture and the organisers used the topic subparts, 'leadership' and 'service', to target relevant campus organisations and departments, like the campus Leadership Development Office, that could help spread the word about the lecture. The organisers also took a close look at the Secretary's biography (http://www.navy.mil/navydata/bios/navybio_ldr.asp?bioID=505) in order to learn more about his background and identify what parts of his background could be used to help build awareness for the speech. They asked themselves, what would make this speaker appealing to different audiences? For instance, while serving as governor of Mississippi Mabus was a strong proponent of education and received recognition for his educational reform; to the organisers, this qualified as appealing information. In requesting the UNC School of Education to share information about this lecture, this part of Mabus's background was mentioned. A sample tweet for the department to post on their account was created and specifically mentioned Mabus' connection to education with the event 
hashtag. A quick search on YouTube showed several videos of the Secretary giving speeches, including one on a naval carrier for a UNC basketball game during the 2011-2012 basketball season. Various tweets were made highlighting these speeches and other parts of his background. This resulted in several RTs, even by the official Twitter accounts for the Johns Hopkins University and the University of Mississippi, two of the schools the Secretary attended.

While all of this information was interesting, it was not, according to the author, in a 'tweetable' format; rather, it was written out in paragraph form. The next step was to convert the biographical paragraphs into short, tweetable snapshots. For example, instead of tweeting "The Secretary of the Navy Ray Mabus will be speaking at UNC on November $1^{\text {st" }}$ the following tweet "@SECNAV Mabus will be speaking at \#UNC on Nov. $1^{\text {st }}$ http://bit.ly/uncmabus \#uncmabus" was sent out. The first version uses only 75 characters while the second uses 83 , but the second tweet provides the event URL, hashtag, and official Twitter handle of the speaker, while the first does not. This change was important because the staff managing Mabus' social media account would see this mention and would be likely to RT it to their followers to further publicise the lecture.

Using official Twitter accounts also proved useful when converting other parts of the Secretary's biography. These official accounts would sometimes RT event organisers' messages to all of their followers, which would increase the impressions for the tweet since most of these official accounts had considerably more followers than the newly established@UNCMPA account. For instance, when talking about the Secretary's law degree, it was suggested that the tweet read “@Harvard_Law grad@SECNAV Mabus to speak at \#UNC on Nov. $1 \mathrm{http} / /$ /bit.ly/uncmabus \#uncmabus". This contains the same information found in the paragraph format biography, but the 'tweetable' form allows for an easier and more shareable format.

Since the Secretary had participated in the UNC Carrier Classic featuring the UNC basketball team, a YouTube video link of his speech at the event was tweeted along with a mention of the UNC campus newspaper. The goal was for the newspaper twitter account to spot this connection between the speaker and the UNC student body, which would increase publicity. In similar fashion, Mabus' environmental focus as Secretary of the Navy created interest in the UNC Environmental Finance Center, while his law background was of interest to the NC Indigent Defense Education program as well as the UNC School of Law and Harvard Law, the Secretary's alma mater. Through a series of tweets, event organisers were able to get the official US Navy, Secretary of the Navy, Johns Hopkins University, and University of Mississippi accounts to RT messages advertising the upcoming speech.

\subsection{Step 4: Compose pre-event and event tweets}

The final week leading up to the event is the point at which the event organiser(s) should spend the most time on social media. At this stage of the process, one has increased the number of followers for the different social media accounts, made different connections, and hopefully created additional interest and excitement for your upcoming event. As the go-live date for the event approaches, the event organiser(s) will want to be sure to continue to tweet. Since the intention is to use the hashtag as a backchannel for the event, one will want to change their tweets to reflect this. Instead of simply tweeting about attending the event, it is a good practice to alert people knows they can follow the lecture using the hashtag. During the actual event, the event organiser(s) tasked with managing 
the social media accounts will want to manage the tweets and respond in a timely fashion to any messages that come into the established social media accounts.

\subsubsection{Populating and engaging the \#uncmabus backchannel}

At this point, the organisers began to recruit others to participate in the social media efforts surrounding the actual lecture. Instead of having only one account or one person live-tweeting, many MPA students and others were asked to tweet the event. While one person would be managing the official account, used for making the live-tweets of the lecture, another would use TweetDeck to monitor mentions or other social media traffic on Twitter about the event. This would allow for maximum engagement in the backchannel.

For this event, the goals were to build buzz for the Secretary's speech, let people know about the lecture, and ensure that the event was well-attended. While there were no tickets for the event and no way to specifically determine which attendees came because of the social media campaign and which showed up due to traditional marketing techniques, the combination of these efforts did contribute to the full lecture hall on the date of the speech. It is worth noting that a link to the hashtagged tweets was added to the SOGs informational page for the lecture, and bit.ly analytics show that during the event six people clicked on the link to follow the lecture through the hashtag. The data only shows the activity of those who clicked the link from the site; it does not show people who may have been following the lecture on their phones or desktops through the hashtag or other tracking methods.

\section{Outcomes}

The author believes that the social media efforts for the Mabus lecture were successful under several evaluation measures. The goal was to create social media buzz, and bit.ly and Crowdbooster statistics shows that this occurred. The planning and execution of the social media campaign spanned one month, from early October to the event date in November. In that timeframe, the @UNCMPA account increased its followers from 7 to 67 and the bit.ly/uncmabus link was clicked on 140 times, with almost half of the traffic coming from Twitter (Figures 3 and 4).

The Crowdbooster dashboard provided some analytical information on the tweets used in the preparation and curation of the backchannel for the Mabus lecture. This data showed that the main UNCMPA Twitter account posted 199 tweets during this one-month period, and this resulted in 41 mentions (roughly a $21 \%$ reply percentage), which is about the average for social media (http://www.sysomos.com/insidetwitter/engagement/). According to the Crowdbooster @UNCMPA dashboard, there were 54 RTs of information originating from the main UNCMPA Twitter account, which garnered 29,605 impressions.

The benefit of Crowdbooster is that one can go back through each tweet made and track its impression rate, thereby identifying the most successful tweets in order to replicate that success with other tweets. This detailed analysis of social media success/failure can be looked at in real-time (making a social media campaign an iterative and adaptive process) or it can be reviewed as part of a post mortem at the conclusion of the campaign (as was done in this case study). In addition to determining top tweets from 
Crowdbooster feedback, one can create a lessons learned document for future events. Apart from Crowdbooster, there are several other sites, some of them free, which can provide analytics. These include TweetStats (http://www.tweetstats.com) and Twitalyzer (http://www.twitalyzer.com), each one of which offers different ways to interpret and calculate the success of your Twitter experience and can provide invaluable information for future adjustments and improvements.

\subsection{Lessons learned}

This was the first time that a coordinated approach was used to connect traditional and social media marketing for an SOG event. In fact, it was the first time that the SOG had ever attempted to use social media in marketing an event, and, accordingly, expectations were purposely kept realistic. For instance, there was no goal to go from 7 to 500 followers in a week or to have 1,000 tweets for this event. Rather given the timeline we had two realistic goals we tried to achieve: we aimed to build awareness of the lecture among multiple audiences and sought to produce a well-attended event.

There were aspects of the marketing process that, if modified, could improve the success of future events. It would have been beneficial, for example, to start the planning earlier. The social media portion of the marketing campaign did not start until October, which left little time to build interest in the lecture. Also, the Twitter account we relied upon had not been used before this event. Now, that the account has been established, however, it should be easier in the future to build interest and traffic for a UNC MPA event. Furthermore, all of our social media efforts were consolidated into a month's time. In the future, it would be optimal to have a longer period for both advertising and engagement around the event. While it is still a best practice to tweet about an event when the event date is near, a longer planning window here would have allowed for a higher level of engagement with both the upcoming lecturer and the intended audience.

To this end, there are several things that can be done for future lectures to better engage the audience and incorporate the backchannel into the lecture, both in advance of the event date and during the speech itself. For example, questions could be solicited from followers for the speaker or even through a moderated, virtual question-and-answer period with the speaker before the lecture. Twitter could also be used to curate questions or topics that people are interested in learning more about from the speaker, and these could be passed on to the speaker in an attempt to incorporate them into the live portion of the lecture.

Social media is a relatively new phenomenon, particularly with respect to conference organisation, and this was evident in this project. The two students who helped with the project had limited experience with Twitter (they had previously used personal, as opposed to professional, Twitter accounts), and neither had any experience with creating a backchannel or working with new hashtags or TweetDeck. A learning curve pertaining to backchannel development happened concurrently with the students' actual development of the backchannel. The author hopes that this case study will provide assistance, context, and an adaptable framework to anyone who is similarly unfamiliar with social media but who wishes to learn more about it and/or create a backchannel. Ferriter (2010) encourages faculty members to experiment with Twitter and become what he terms 'efficient digital learners' who can, in turn, deploy Twitter as a tool within their own classrooms (p.74). The author approves of this approach and hopes it will be embraced by more instructors. 
Twitter is proving to be a great tool for starting and engaging in meaningful conversations and for providing information to the masses. Researchers are beginning to develop ways to curate and mine Twitter data. The CDC, for example, has used tweets to try to estimate flu epidemics and to measure public sentiment on other pressing health issues (Savage, 2011). As Twitter becomes more widely used and adaptable, the opportunities for tapping into the rich data it produces will continue to evolve.

\section{Conclusions}

The concepts and best practices used by the average citizen to grow a following and crowd source ideas (that is, obtain ideas by soliciting thoughts from large groups of people, particularly online) can

1 be adapted to build an event backchannel

2 have applications in higher education and in conference organising generally.

The first step in translating these tactics over involves learning what tools are available. One would then select the approach that best fits the specific needs of his or her project (e.g., backchannel development, classroom instruction, event marketing). As Twitter expertise grows among members of the public, and as methods for tracking and analysing its effectiveness similarly increase, we should be able to find new and interesting ways to implement this new communication tool to enhance overall learning and engagement.

The purpose of this article was to provide event organisers with an easy-to-follow, step-by-step guide for creating an event backchannel. In a relatively short period of time, the UNC SOG was able to create enough buzz around a lecture to generate over 25,000 impressions on Twitter (measured by tracking only the School's official Twitter account and not including all of the other personal accounts that were simultaneously sending out messages and creating additional traffic during the event). The hope is that readers will use our experience to create their own social media successes.

Social media is commonly perceived as the 'low-hanging fruit' in marketing circles because it can be highly effective yet has a low threshold for entry and execution. Despite this ease of use, it is crucial that careful social media planning be done to ensure a successful event. The most important factor for achieving backchannel success (or Twitter success generally) involves the rate of engagement with followers and with the information being shared. People will follow and engage with an account that is providing good information or some other benefit, and higher rates of engagement will yield higher rates of shares, mentions, RTs, and followers.

\section{Acknowledgements}

The author would like to thank Jean Coble, Gini Hamilton (MPA Programme and Marketing), Jamar Jones, Kevin Justice, Melissa Twomey, Stefanie Panke (Publications and Instructional Support), Katie Paulson, Priyanka Vakil and Trey Mangum (MPA students and work study students) for their invaluable assistance on this project and production of this paper. The author would also like to acknowledge the generous support of Fidelity Investments and the MPA Alumni Association in sponsoring the lecture. 


\section{References}

Bakshy, E., Hofman, J., Mason, W. and Watts, D. (2011) 'Everyone's an influencer: quantifying influence on Twitter', Proceedings of WSDM '11, Hong Kong, China.

Ebner, M., Muhlburger, H., Schaffert, S., Schiefner, M., Reinhardt, W. and Wheeler, S. (2010) 'Getting granular on Twitter: tweets from a conference and their limited usefulness for non-participants', in A. Reynolds and M. Turcsanyi-Szabo (Eds.): Key Competencies in the Knowledge Society, pp.102-113, Springer, Berlin Heidelberg.

Ferriter, W.M. (2010) 'Why teachers should try Twitter', Educational Leadership, Vol. 67, No. 5, pp.73-75.

Harrington, S., Highfield, T. and Bruns, A. (2012) 'More than a backchannel: Twitter and television', Noguera, J.M. (Ed.): Audience Interactivity and Participation, pp.13-17, COST Action ISO906, Transforming Audiences, Transforming Societies, Brussels.

Highfield, T., Harrington, S. and Bruns, A. (2013) 'Twitter as a technology for audiencing and fandom', Information, Communication \& Society, Vol. 16, No. 3, pp.315-339.

McNely, B. (2009) 'Backchannel persistence and collaborative meaning-making', Proceedings of the 27th ACM International Conference on Design of Communication, Bloomington, IN, 5-7 October, pp.297-303.

Murthy, D. (2011) 'Twitter: microphone for the masses?', Media, Culture \& Society, July, Vol. 33, No. 5, pp.779-89, doi:10.1177/0163443711404744.

Qualman, E. (2013) Socialnomics: How Social Media Transforms the Way We Live and Do Business, 2nd ed., John Wiley \& Sons, Inc., New Jersey.

Morville, P. and Rosenfield, L. (2002) Information Architecture for the World Wide Web, 2nd ed., O'Reilly \& Associates, Inc., California.

Ross, C., Terras, M., Warcik, C. and Welsh, A. (2011) 'Enabled backchannel: conference Twitter use by digital humanists', Journal of Documentation, Vol. 67, No. 2, pp.214-237.

Salesforce Marketing Cloud (2012) Strategies for Effective Tweeting: A Statistical Review [online] http://www.salesforcemarketingcloud.com/resources/ebooks/ strategies-for-effective-tweeting-a-statistical-review/ (accessed 20 April 2013).

Savage, N. (2011) 'Twitter as medium and message', Communications of the ACM, Vol. 54, No. 3, pp.18-20, doi:10.1145/1897852.1897860. 